\title{
Challanging Management of a Giant Sublingual Dermoid Cyst Rapidly Enlarged Throughout Pregnancy and Influence of Hormonal Factors
}

\author{
Massimo Mesolella*, Elena Cantone, Vieri Galli, Maurizio Iengo \\ ENT Department, University Federico II of Naples, Naples, Italy \\ Email: "massimo.mesolella@tin.it
}

Received January 8, 2013; revised February 11, 2013; accepted February 19, 2013

\begin{abstract}
The authors present a case with a sublingual giant dermoid cyst, rapidly enlarged during pregnancy, which showed itself with deglutition and mild respiratory problems. Dermoid cysts (DCs) are benign lesions arising from entrapment of epithelial rests during embryogenesis. They are most frequently located in the ovaries and testes. Up to $6.9 \%$ are found in the head and neck and $1.6 \%$ within the oral cavity, representing less than $0.01 \%$ of all oral cavity cysts. They usually present early in life as asymptomatic masses with slow and progressive growth. However, they may reach a large size with a potential impact on deglutition, speech, and respiration. It is suggested that the rapid growth of DCs during pregnancy is associated with the increase of plasma levels of estrogens and progesterone. In order to eliminate respiratory and deglutition issues, it was decided to perform the surgical excision after a cesarean delivery at 29th week.
\end{abstract}

Keywords: Dermoid Cyst; Sublingual Mass; Airway Obstruction; Pregnancy; Estrogens; Progesterone

\section{Introduction}

Dermoid cysts (DCs) are benign lesions arising from entrapment of epithelial rests during embryogenesis [1]. They are most frequently located in ovaries and testes. Up to $6.9 \%$ are found in the head and neck and $1.6 \%$ within the oral cavity representing less than $0.01 \%$ of all oral cavity cysts [2]. They usually present early in life as asymptomatic masses with slow and progressive growth. However, they may reach a large size, becoming clinically apparent in adolescents and young adults, with a potential impact on deglutition, speech, and respiration [3].

Sublingual DCs may develop above or below the mylohyoid muscle, causing a different clinical presentation. In the first case, DCs produce a sublingual mass in the floor of the mouth which push the tongue upwards and backwards. In the latter case, instead, DCs produce a well-circumscribed, submental mass, just beneath the skin.

It has been suggested that DCs increase in size after puberty because of hormonal changes that stimulate the sebaceous glands these tumors contain. Additionally, it was proved that growth rate of DCs in premenopausal women was significantly higher than that in postmenopausal women [4].

${ }^{*}$ Corresponding author.
The treatment of choice is surgical excision. An intraoral incision may be used for small cysts, but large ones require an external approach which avoids intraoral contamination and allows better visualization and control surrounding structures [5]. In this case report, it is presented a case with a sublingual giant DC, first noticed just after menarche, and rapidly enlarged during pregnancy, which showed itself with deglutition and mild respiratory problems. There was only one similar case described in literature [6].

\section{Case Report}

A 27-year-old woman at her 26th week of gestational age was admitted to our clinic complaining a giant tumor in sublingual region. She first noticed the existence of an asymptomatic mass at age of $14 \mathrm{yrs}$, after menarche, which did not grow significantly until pregnancy. The mass, rapidly growing throughout pregnancy, caused dysphagia and dyspnea in supine position.

On examination, there was a $10 \mathrm{~cm}$ sublingual mass of soft consistency filling the floor of the mouth and pushing the tongue upward toward the palate (Figure 1). Biochemical laboratory data were normal, except for an increase of liver enzymes. Ultrasonography showed a 67 $\times 75 \mathrm{~mm}$ hypoecogenic cystic mass, with well defined margins and regular walls. 


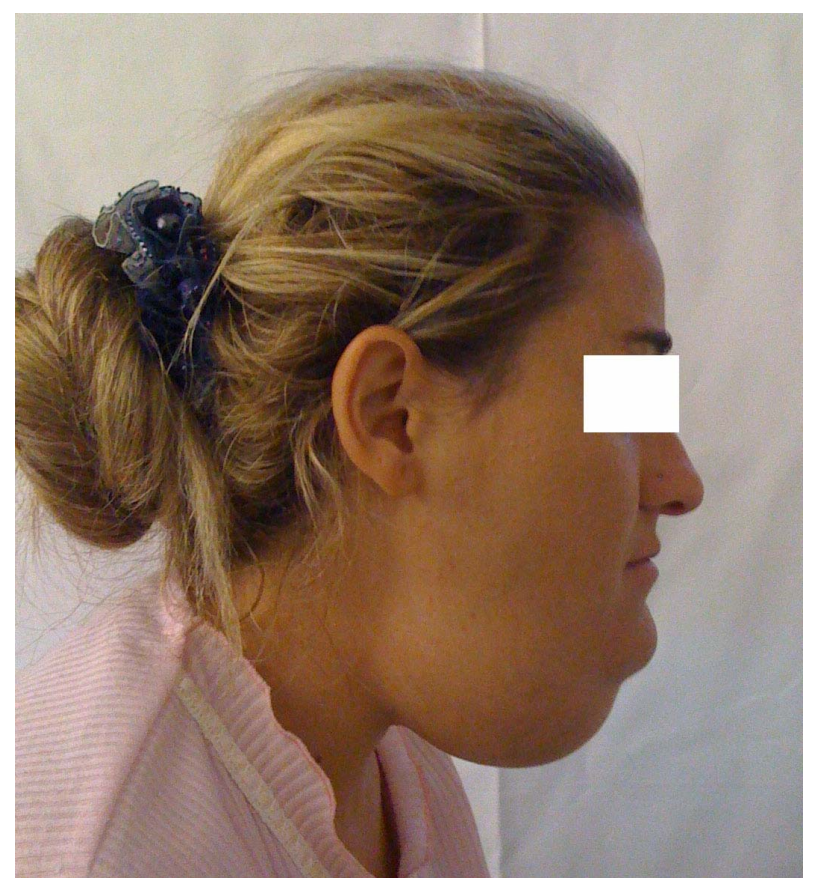

Figure 1. At neck examination, a midline, non tender, soft, $10 \mathrm{~cm}$ diameter tumor occupied the sub mental space but was partially movable.

Two weeks later, ultrasonography demonstrated a $96 \times$ $77 \mathrm{~mm}$ mass. The content of the cyst was heterogeneous. A magnetic resonance imaging scan revealed a $96 \times 85 \times$ $71 \mathrm{~mm}$ well-circumscribed non-enhancing cystic mass extending from the jaw to the base of the tongue, disloting epiglottis and palatine tonsils, extending inferiorly through geniohyoid, genioglossus muscles and between submaxillary glands, hyoglossus and mylohyoid muscles. The inferior border of the tongue was compressed and the tongue was dislocated superiorly (Figure 2). The enlargement occurred despite treatment with antibiotics. In the first time it was decided to aspirate the fluid cont in the cyst, in order to delay the potential life threating surgery. Unfortunately, the high-density fluid content of the cyst prevented from the full aspiration. The small amount which was aspired was not conclusive.

The worsening of clinical symptoms, such as dysphagia and dyspnea, and gestational age of the fetus (29 weeks) considered safe for the health of the fetus itself, persuaded us to perform both operations at the same time: before cesarean section and after he cyst exeresis.

It was considered that an extra-oral approach would be more appropriate than an intraoral route, under general anesthesia delivered by nasotracheal intubation without tracheotomy.

A horizontal submental incision was performed; the deep fascia was entered to reveal a well-encapsulated cystic mass. The wall of the cyst was thin and regular, and the contents consisted of homogenous fluid. Micro- scopic examination showed a cyst lined by keratinizing stratified squamous epithelium, sebaceous glands consistent with dermoid cyst. The immunohistochemistry showed a high concentration of both estrogens and progesterone receptors (Figure 3). There was no evidence of recurrence at 2 years after treatment. This case report was validated by the institutional review board of this faculty.

\section{Discussion}

Dermoid cysts that derived from pluripotential of ectodermal origin, are congenital slow-growing lesions generally diagnosed in young adults in the second and third

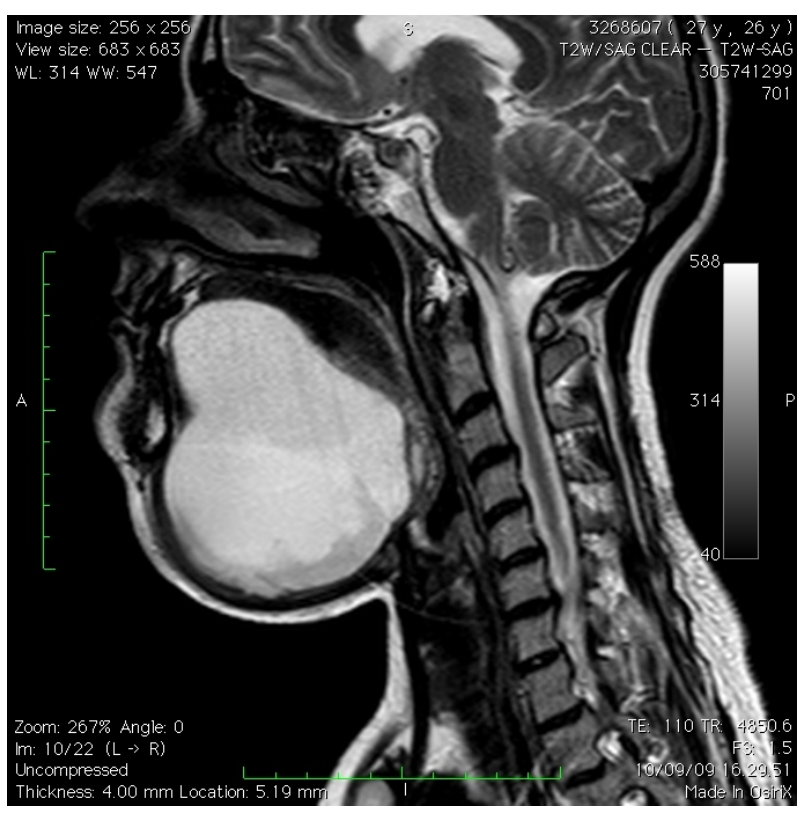

Figure 2. MRI of the neck in both axial and coronal sections, without contrast, revealed a nodular heterogenic tumor in midline that occupied the sublingual space, which dimension was $96 \times 85 \times 71 \mathrm{~mm}$ (height $\times$ depth $\times$ width).

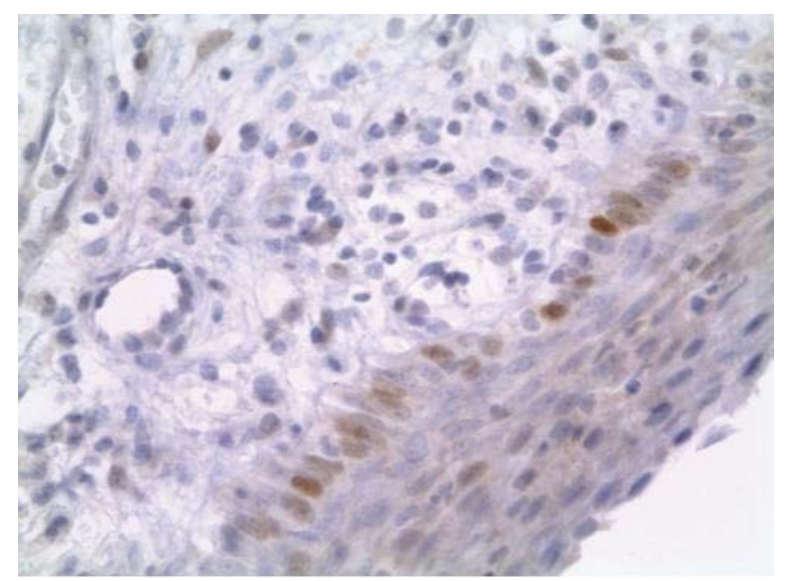

Figure 3. The immunohistochemstry showed a high concentration of both estrogens and progesterone receptors. 
decades of life. Median floor of mouth occur Sublingual DCs are uncommon. Because they can displace the tongue, patients usually present with dysphagia, dysphonia, and dyspnea.

Differential diagnosis of swellings of the neck and floor of the mouth, include slipomas, Ludwig's angina, acute infection or cellulitis, ranula, thyroglossal duct cyst, cystic hygroma, unilateral or bilateral blockage of Wharton's ducts, branchial cleft cysts, infection of submaxillary and sublingual glands, and benign and malignant tumors of the floor of the mouth and adjacent salivary glands [7].

The exact mechanism of the growth of DCs is unknown, it has been suggested that ovarian DCs increase in size after puberty because of hormonal changes that stimulate the sebaceous glands that these tumors contain [6].

The presence of sublingual dermoid cyst women during pregnancy is a potential risk for both the mother and the fetus because of the susceptibility to ovarian hormones increase in size of the mass. Almost all hormones, (estrogens, progesterone, thyroid, parathyroid, and cortisole) levels elevate in the plasma during the gestational period.

Also Papadogeorgakis [7] observed in his case report that gross enlargement occurred after recent pregnancy.

We suggest that the rapid growth of sublingual dermoid cyst during pregnancy, is associated with the increase of plasma levels of estrogens and progesterone, acting as growing factor for the cyst, which expresses an increased number of hormonal receptors. This theory was validated with the immunohistological study of the surgical sample, which showed in our patient an increased concentration of both estrogens and progesterone receptors (Figure 3).

Tuz [6] reported a case with a sublingual giant dermoid cyst growing during pregnancy, but the patient have manifested mild respiratory and deglutition problems so the Authors proposed the removal of cyst before attempting to conceive in order to eliminate the risk of rapid growth. Surgical procedure was done under general endotracheal anesthesia.

In our case the patient presented to the emergency room complaining of breathing difficulties and inability to eat or to talk. Because of the large size of the cyst, extraoral approach was performed before attempting to conceive in order to eliminate the consequences related to the fetus.

A multidisciplinary approach is required, including ENT surgeons, anesthesiologists, pediatric and gynecologists.

\section{Conclusion}

Congenital cysts, either dermoid or teratoid, of the sublingual region are rare entities. Their presence in women is a potential risk for both the mother and the fetus during pregnancy, because of the susceptibility of the dermoid cyst to ovarian hormones such as progesterone and estrogens acting as growing factor for the cyst, which expresses an increased number of receptors. Therefore, it is suggested the exeresis of the DC since puberty.

\section{REFERENCES}

[1] M. Yilmaz, H. Vayvada and C. Demirdover, "Dermoid Cyst at the Suprasternal Notch,” Annals of Plastic Surgery, Vol. 45, No. 3, 2000, pp. 343-345. doi:10.1097/00000637-200045030-00027

[2] S. Fuchshuber, G. Grevers and W. J. Issing, "Dermoid Cyst of the Floor of the Mouth-A Case Report," European Archives of Oto-Rhino-Laryngology, Vol. 259, No. 2, 2002, pp. 60-62. doi:10.1007/s004050100413

[3] W. S. Gibson Jr. and N. A. Fenton, "Congenital Sublingual Dermoid Cysts,” Archives of Otolaryngology—Head \& Neck Surgery, Vol. 108, No. 11, 1982, pp. 745-747. doi:10.1001/archotol.1982.00790590067018

[4] V. Rajayogeswaran and J. W. Evenson, "Epidermoid Cyst of the Buccal Mucosa," Oral Surgery, Oral Medicine, Oral Pathology, Vol. 67, No. 2, 1989, pp. 181-184. doi:10.1016/0030-4220(89)90326-5

[5] D. Bloom, D. Carvalho, J. Edmonds, et al., "Neonatal Dermoid Cyst of the Floor of the Mouth Extending to the Midline Neck," Archives of Otolaryngology_Head \& Neck Surgery, Vol. 128, No. 1, 2002, pp. 68-70.

[6] M. Tuz, H. Dogru, K. Uygur and B. Baykal, "Rapidly Growing Sublingual Dermoid Cyst throughout Pregnancy," American Journal of Otolaryngology, Vol. 24, No. 5, 2003, pp. 334-337. doi:10.1016/S0196-0709(03)00051-6

[7] N. Papadogeorgakis, E. F. Kalfarentzos, C. Vourlakou and C. Alexandridis, "Surgical Management of a Large Median Dermoid Cyst of the Neck Causing Air Way Obstruction. A Case Report," Oral and Maxillofacial Surgery, Vol. 13, No. 3, 2009, pp. 181-184. doi:10.1007/s10006-009-0165-5 